\title{
Soft Haptic Interface based on Vibration and Particle Jamming
}

\author{
Joshua P. Brown and Ildar Farkhatdinov
}

\begin{abstract}
Whilst common in devices ranging from smartphones to game controllers, vibrotactile feedback has generally been limited to providing a uniform sensation across a tactile surface. We propose a haptic interface based on the emerging physical effect of particle jamming with both vibrotactile and shape changing outputs, which can be extended in space to create haptic surfaces and devices with shape and vibrotactile responses localised to one part of the device. This paper gives an overview of the physical principles behind this technology and presents detailed performance metrics obtained from a working prototype. These include experimental characterization of the relationships between air pressure and electric motor power and vibration amplitude and frequency which show that it is possible to control vibrotactile amplitude and frequency independently.
\end{abstract}

\section{INTRODUCTION}

Particle jamming, the physical effect of compacting, and thus stiffening, a fluidic body of particles is a rapidly emerging technology, already being employed in haptics and robotics. The body of particles can be made to react differently to physical stimuli such as a deliberate and forced change in its volume or a user's touch, making it possible to dynamically control a variety of tactile sensations.

The jamming of particles in a fluid is a physical phase transition [1] and a particulate fluid whose particles are packed in such a way that they are connected to all adjacent particles can be said to be in jammed state. Jammed fluids are slightly denser than the same material in a loose or unjammed state due to their inherently lower volume and this leads to many changes in their properties, such as increased viscosity and stiffness caused by mechanical support between the particles.

Particle jamming has been used to create small and large scale tactile interfaces. For example, soft cells filled with small particles can be hardened by creating an area of low air pressure inside an arrangement of the cells, affecting their stiffness and by extension, resistance to the user's touch [2], [3], [4], [5], [6]. Large format display based on particle jamming was used to transmit vibration uniformly to a large body areas [7]. Particle jamming has also been applied to wearable robotic devices to provide kinesthetic feedback [8]. To some extent, the particle jamming based haptic interface

This project has received funding from the IEEE RAS Technical Committee on Haptics under the "Innovation in haptics" research programme. The authors express their thanks to the committee for their support.

Additional support was provided by the UK EPSRC grant NCNR $\mathrm{EP} / \mathrm{R} 02572 \mathrm{X} / 1$.

Both authors are with the School of Electronic Engineering and Computer Science, Queen Mary, University of London, Mile End, London, UK $\{j \cdot$ p.brown, i.farkhatdinov\}@qmul.ac.uk

I. Farkhatdinov is also with the Department of Bionegineering of Imperial College of Science, Technology and Medicine, London, UK.

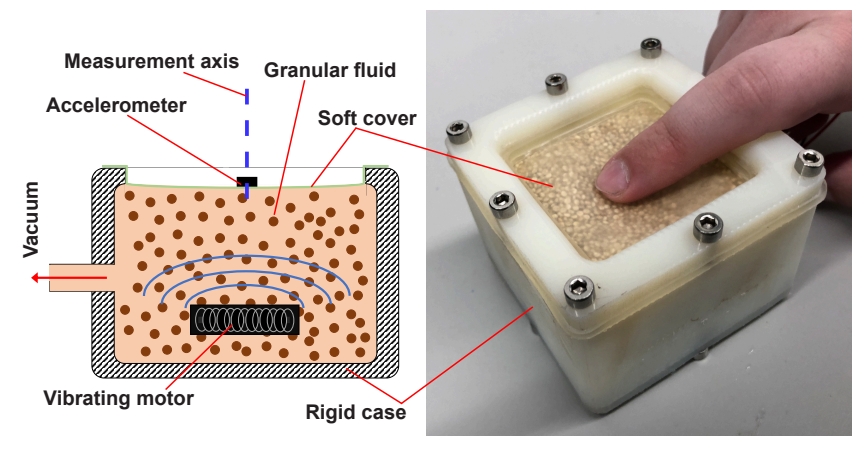

Fig. 1. Left: Physical schematic showing the key components of the jamming device. Right: A simple prototype device consisting of a single jamming cell.

can behave similarly to liquid based interfaces. Past work has included a magnetically driven fluid display, where areas of a MHF fluid corresponding to meaningful shapes are stiffened by magnetic coils [9].

A prototype device (Fig. 1 right) has been constructed to answer a number of research questions prior to integrating the particle jamming technology into interactive haptic devices. These are:

1) What is the effect on the vibrotactile response of modulating the stiffness of the particle fluid?

2) What is the effect on the vibrotactile response of modulating the power supplied to the vibrating motor?

3) What is the effect on the vibrotactile response of a user physically interacting with the device?

One question not being asked here is the effect on vibrations felt on the rigid parts of the device. This is because the testbench being used here has rigid components that will not exist when the technology is integrated into HCI devices (in the case of a wearable, there may be no rigid components). This response will be investigated when the particle jamming system is applied to other devices.

\section{Haptic Interface BASEd on PARTicle JAMMing}

This work proposes that a controllable stiffness fluid consisting of small particles can be used to control the uniform haptic vibrations produced by a motor. This effect can be achieved by reducing the air pressure in the particles' container, causing the soft haptic pad to jam them together. This jamming effect causes the body of particles to stiffen, decreasing the motor's capacity to displace itself within the fluid, and thus the amplitude of the vibrations transferred to the finger pad. By releasing the low pressure, the fluid returns to a free state, allowing the motor to move vibrate strongly again. Conversely, increasing the air pressure inside 


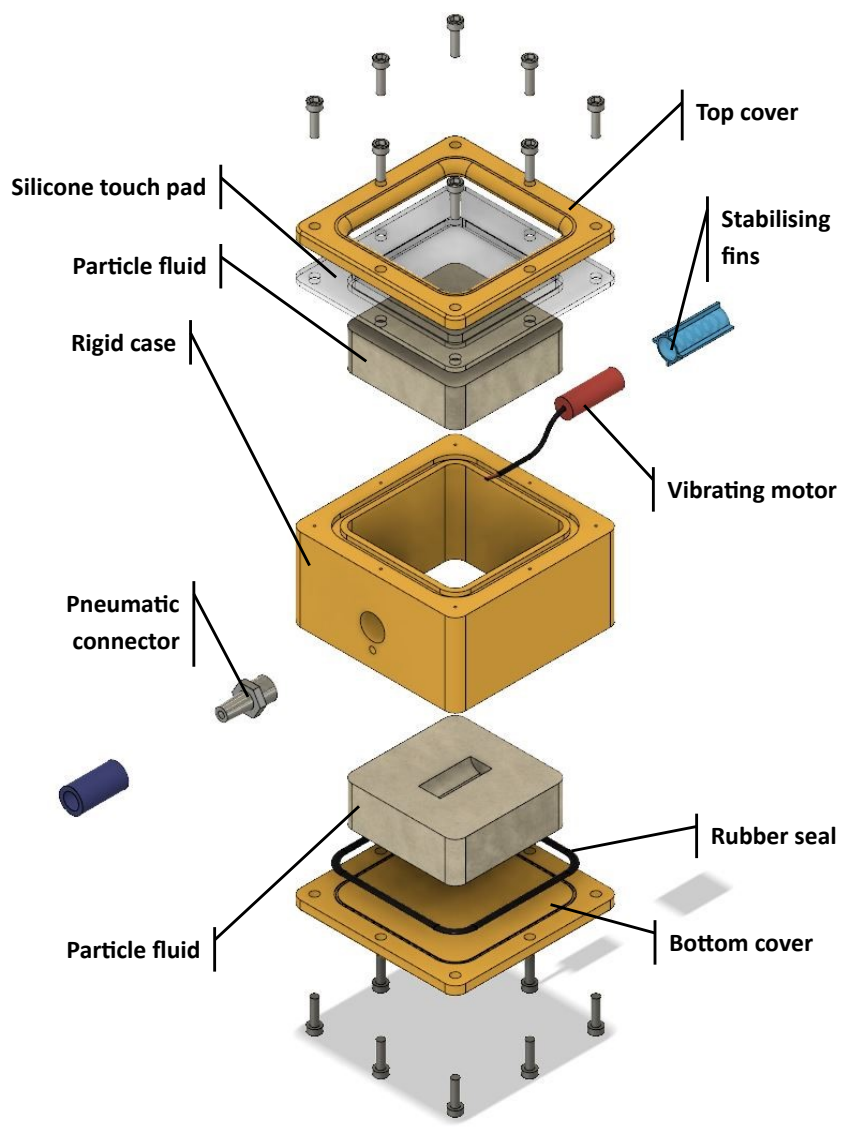

Fig. 2. The assembly structure and components of the prototype haptic interface

the device will cause the soft pad to inflate and create a shape upward. This will also have the effect of very suddenly reducing the vibration intensity as there will no longer be a strong contact between the vibrating particle body and the user's finger.

As an initial prototype to evaluate this idea, one module was produced to enable an experimental study of the effect of particle jamming on vibrations. This comprises a rigid box measuring 80x80x50mm made from milled Nylon which holds $75 \mathrm{~g}$ of the particulate fluid, in this case Quinoa seeds of about $1 \mathrm{~mm}$ in diameter. This is seasled with a soft silicone touch pad. A Precision Microdrives 307-100 fully encapsulated 9mm ERM (Eccentric Rotating Mass) vibrating motor was suspended in this container to generate the vibrations, but loosely tethered between two opposite sides so as to control the experimental conditions. This allows over $10 \mathrm{~mm}$ of movement on all sides, but does not allow the motor to move too far from the centre of the box to affect the measurement results. This can be connected either to a power supply or a computer controlled driver. The ERM motor was chosen as a vibrating source as it offers a better maximum amplitude than voice coils and its output is less directional than an LRA (Linear Resonant Actuator) which is important for observing superposition effects inside the particle fluid. A

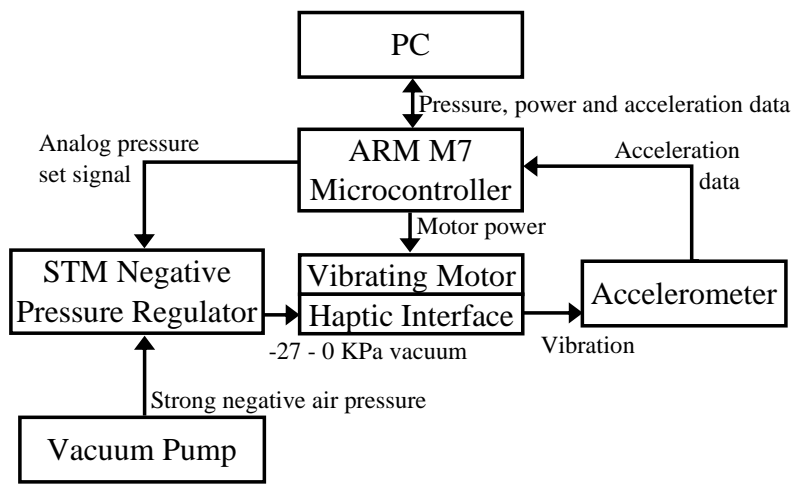

Fig. 3. The complete control and measurement system

mesh filter and $4 \mathrm{~mm}$ push fit connector allow the prototype unit to be connected to a pneumatic control system. A layer of soft foam is used inside the case to speed up the loosening of the particles when the vacuum is released (Fig. 2).

Haptic feedback is presented via a $1 \mathrm{~mm}$ thick silicone pad. This was 3D printed with a Formlabs Form 2 laser 3D printer in order to set the touch pad into the box to improve the vacuum seal under vibration. This process will also allow textures to be added for future psycophysical experiments. This presses against the particle body under negative air pressure and transfers vibration to the user, but can also be inflated to present shape with high positive air pressure. The shape display will form part of another study.

The low pressure in the device is achieved with a $750 \mathrm{~W}$ vacuum pump and controlled by an STM 0090-3 series electronic negative pressure regulator. The regulator also features readout from a pressure sensor which enables accurate measurement of the pressure inside the particle fluid.

\section{EXPERIMENTAL PROCEDURE}

The particle jamming system described above differs from many existing vibrotactile actuators in that it can be controlled by varying both the air pressure within the particle fluid, but also the electronic signal sent to the vibrating motor. Understanding the effect of modulating each control signal (and both together) is an important first step in understanding the characteristics of the system.

\section{A. Range of vibrotactile response}

As an initial exercise, the two input signals were cycled from their minimum to their maximum input values. For the air pressure, this can be set anywhere down to - $24 \mathrm{KPa}$ (the lowest pressure at which the vibrations are known to be affected based on a previous pilot study) and for the motor speed, this is a supply voltage up to the rated maximum of 3.3V. This method of control was adopted over a more conventional pulse-width-modulation approach in order to ensure that any unusual features in the recorded vibrations were not related to instability in the power supply. Decreases in air pressure were made at approximately $1 \mathrm{kPa}$ intervals, whilst the motor voltage was increased in steps of $0.1 \mathrm{~V}$. 
Steady-state (ignoring transients) vibrations that would be transferred to a user were measured by a $3 \times 3 \mathrm{~mm}$ MEMS accelerometer (Analog Devices ADXL-337) which was glued to the surface of the touch pad so that the $\mathrm{z}$-axis was aligned perpendicular to the centre of the touch surface (Fig. 1 left). This measured the acceleration of the tactile pad with a sampling rate of $27 \mathrm{KHz}$. As acceleration is measured, it is important to note that all results will have a bias of $9.81 \mathrm{~ms}^{-2}$ due to gravity. Amplitude of vibration is given as the peak-peak acceleration, calculated for each vibration as an average of the peak-peak acceleration for each cycle (as in Fig. 4).

\section{B. Electro-pneumatic control}

Following on from this, understanding and quantifying the extent to which input vacuum pressure and motor speed can affect the amplitude and frequency of felt vibrations is important in understanding the system model and designing a control scheme. This part of the investigation will refer to additional data collected above but analyse this in greater depth to allow meaningful relationships to be identified between the pairs of input and output quantities.

\section{Response to mechanical interaction}

In real world use cases, it is entirely likely that users will exert some force on the touch surface and, by extension, the body of particles underneath. The natural hypothesis is that this will have the effect of manually actuating the jamming action of the particles. In this experiment, the prototype interface was set to vibrate under constant control signals, whilst a finger pressed down on the touch surface with a force measured by a weighing scale underneath the setup. This experiment captured fingertip force response data at seven discrete force levels, each acting on the particle fluid in soft (minimal vacuum), medium and hard (-27KPa) configurations.

\section{RESUlts}

\section{A. Baseline performance of the vibration actuator}

It is important to first quantify the performance of the vibrating source so as to be able to draw robust conclusions about the effectiveness of the particle jamming system. From the test described above, it was determined that when powered by a $3.3 \mathrm{~V}$ supply the source motor vibrates with an amplitude of $40 \mathrm{~ms}^{-2}$ and at frequencies slightly above $200 \mathrm{~Hz}$. This is well inside the range of human perceptible tactile vibrations [10] and in line with the manufacturer's specifications. This involved attaching the MEMS accelerometer to a free-hanging ERM motor such that the X-Axis was aligned along the diameter of the rotation of the eccentric mass.

\section{B. Range of vibrotactile response}

The first useful result from this programme of work is an indication of the range of vibrotactile sensations that the particle jamming system can provide. These were plotted as a

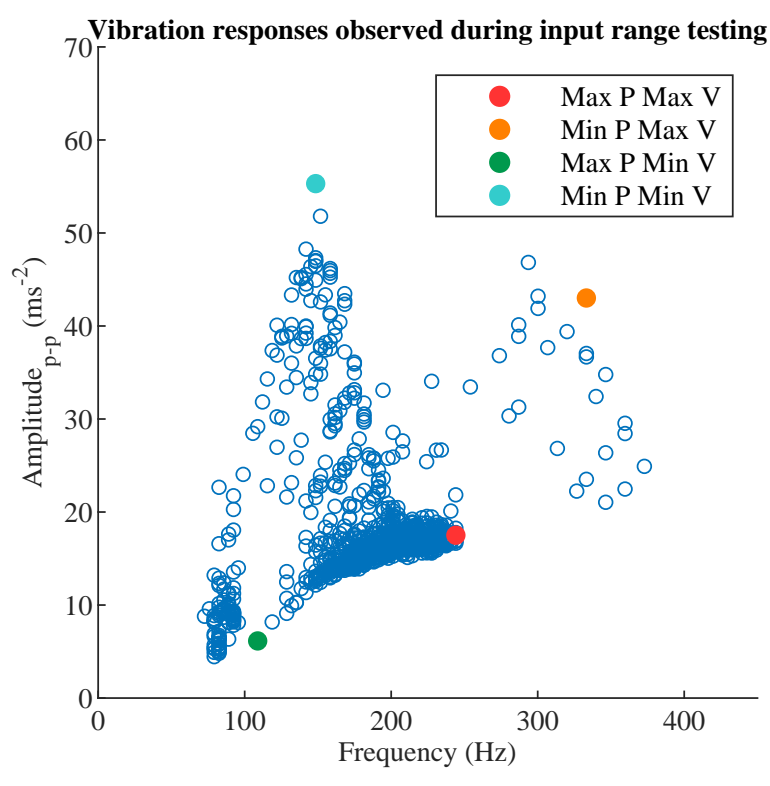

Fig. 4. Vibrotactile signals observed from the prototype interface $(\mathrm{P}=$ pressure, $\mathrm{V}=$ motor voltage)

frequency-amplitude map to give a very quick interpretation of the achievable vibration outputs.

This shows that the prototype device is capable of delivering vibrations of amplitudes between 4.5 and $55 \mathrm{~ms}^{-2}$ and frequencies between 80 and $370 \mathrm{~Hz}$. The distribution of observed samples also shows significant control redundancy in the low amplitude, low-medium frequency part of the range, meaning that a many different control inputs can create the same output. This is to be expected as both low motor drive and high vacuum pressure can reduce the amplitude of vibrations. Conversely, the high amplitude regions of the graph feature fewer samples, though we propose that a closed-loop control system would be able to reach any vibrotactile response within the range of observed vibrations. There were very few vibration recordings that would be classed as outliers, though the middle of the frequency range is somewhat sparsely populated at high amplitudes (Fig. 4).

\section{Effect of vacuum pressure on vibration response}

The first characteristic of the particle jamming system that can be controlled is the vacuum pressure applied to the particle fluid. Intuitively, stiffening the fluid should restrict the vibrating motor's freedom to move, so there should be an observable reduction in amplitude at higher vacuums.

This intuition is borne out by the experimental observations, which show that the amplitude of vibration drops from $50 \mathrm{~ms}^{-2}$ to $20 \mathrm{~ms}^{-2}$ over the range of pressures used in the experiment. Another interesting observation is that the shape of the vibration waveform has a noticeable double peak in the soft fluid, but becomes smoother quite rapidly as the fluid stiffens (Fig. 5).

The frequency of vibration is also affected by the state of the particle fluid, with the dominant frequency of vibration 

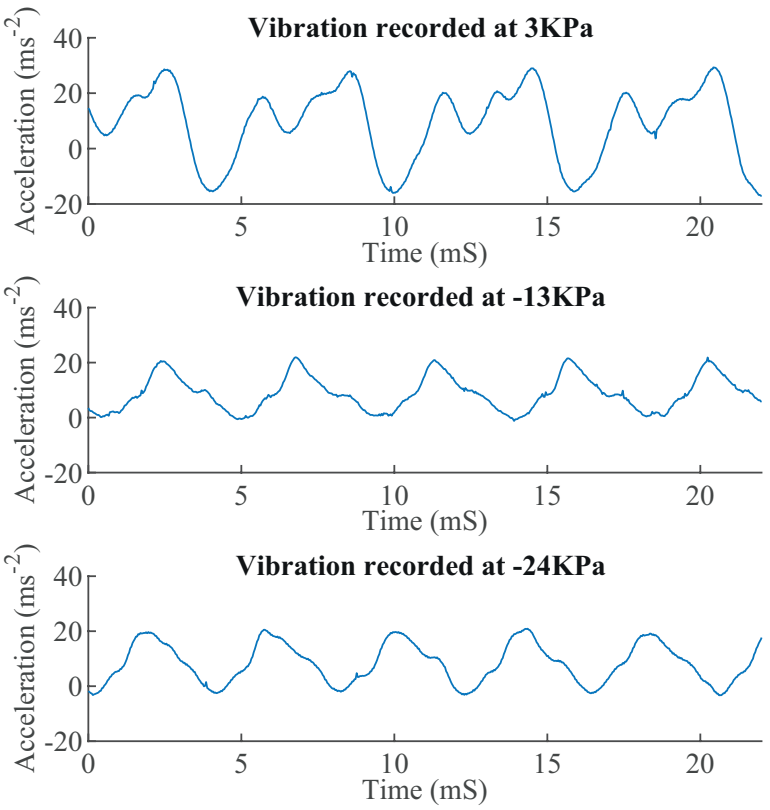

Fig. 5. Vibrations recorded at various pressure levels

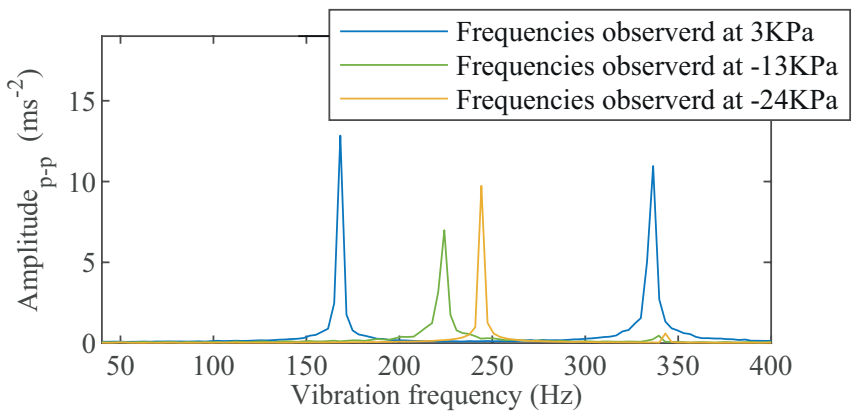

Fig. 6. Frequencies of vibrations recorded at various pressure levels

shifting from $148 \mathrm{~Hz}$ to $230 \mathrm{~Hz}$ over the full range of vacuum pressures (Fig. 6). This is a significant shift but only represents a fraction of the overall frequency response of the prototype device, as ascertained in the experiment above.

\section{Effect of motor speed on vibration response}

The second control input parameter is the power supplied to the vibrating motor.

Testing showed that under low vacuum pressure, and thus with the particles in their soft state, increasing motor power created a more pronounced periodic vibration and a slight increase in amplitude (Fig. 7). Frequency remained fairly consistent under this condition.

Under a strong vacuum, and with a rigid particle body, the increasing motor power doubles the amplitude of vibration up to about $50 \%$ power, at which point amplitude remains consistent. Frequency does however change substantially throughout the range of motor powers, indicating that in a future control scheme motor power could be used to effect a substantial shift in vibration frequency whilst having little impact on amplitude, thus decoupling these two ordinarily related quantities (Fig. 8).

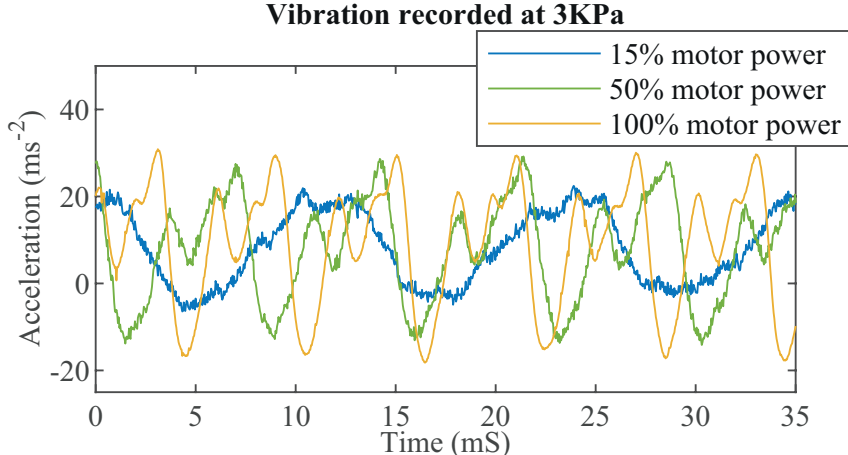

Vibration recorded at $-24 \mathrm{KPa}$

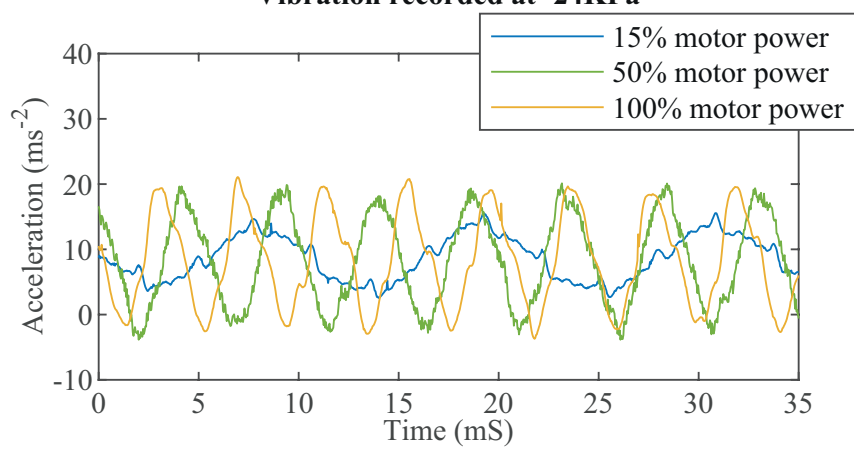

Fig. 7. Vibrations recorded under several levels of vibration motor voltage
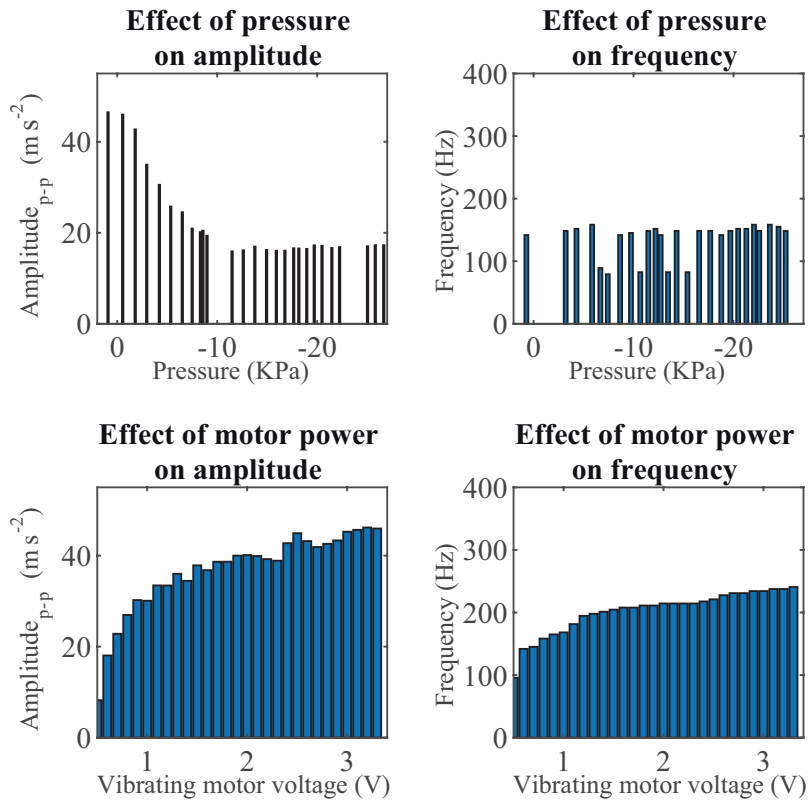

Fig. 8. Relationship between control inputs and vibration parameters under full motor power (pressure control) or $3 \mathrm{KPa}$ vacuum (motor control) 
Vibration recorded at $3 \mathrm{KPa}$

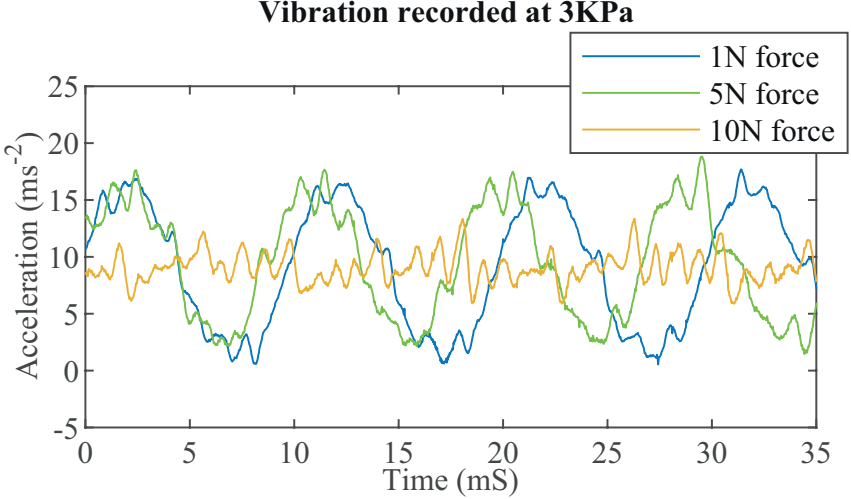

Vibration recorded at $-24 \mathrm{KPa}$

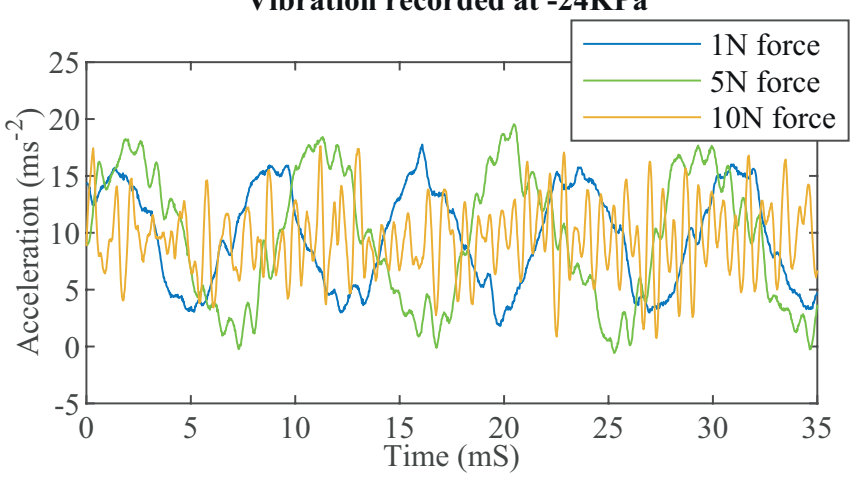

Fig. 9. Vibrations recorded under different levels of fingertip pressure and vacuum strength

\section{E. Effect of external mechanical forces}

Mechanical forces, such as those exerted by a user's hand or force-feedback system, have a substantial effect on the shape, though much less so on the overall amplitude of vibrations observed from the particle jamming system.

Under minimal vacuum, when the interface is still relatively soft, there is a pronounced change from a relatively smooth signal with well defined periodicity, to a very erratic vibration, though there is still an observable periodicity to this signal (approximately $0.5 \mathrm{uS}$ under $10 \mathrm{~N}$ fingertip force). The overall amplitude of the observed vibration is approximately halved from around $20 \mathrm{~ms}^{-2}$ to slightly over $10 \mathrm{~ms}^{-2}$ at the largest peaks. Amplitude remains relatively stable at low fingertip forces, with no significant change in the first half of the measured range $(1-5 \mathrm{~N})$.

The frequency spectra of the vibrations under mechanical force demonstrate this effect, with the peak frequency component shifting only very slightly up to $5 \mathrm{~N}$ of applied force, but then changing dramatically with additional frequency components (which are not harmonics of the first) showing as equally significant parts of the overall signal. It will be down to a future user study to determine the perceptual effect of these changes.

Similar results are observed under high vacuum pressure $(-24 \mathrm{KPa})$ when the particle fluid is already very stiff. In this case, there is a negligible change in overall amplitude $\left(20 \mathrm{~ms}^{-2}\right.$ between peaks is maintained consistently) but the deterioration of the smooth, periodic vibration is more pro-
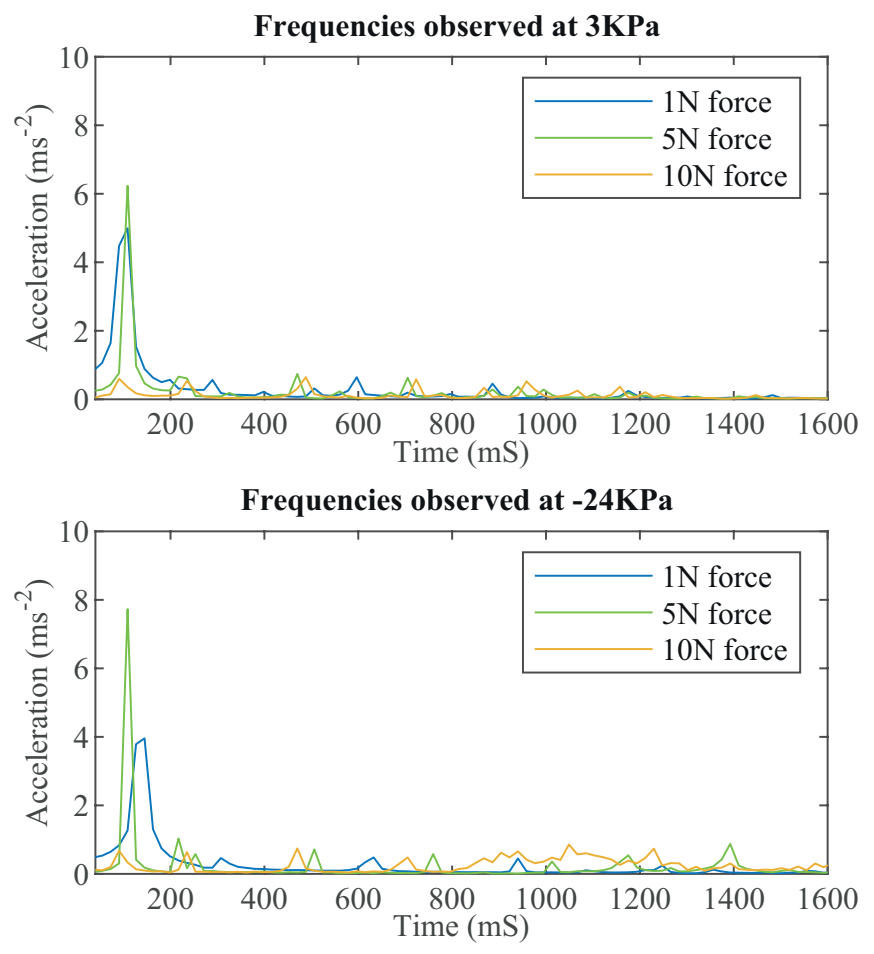

Fig. 10. Frequencies observed under different levels of fingertip pressure and vacuum strength

nounced, with perturbations apparent under forces as low as $2 \mathrm{~N}$. Observable periodicity is completely lost under forces above $8 \mathrm{~N}$ (Fig. 9).

This is apparent from the frequency spectrum which again shows a number of other frequency components below $700 \mathrm{~Hz}$, but also much higher frequencies between 800 and $1400 \mathrm{~Hz}$ (Fig. 10).

\section{Discussion ANd Future Work}

Whilst this technology was initially intended to use air pressure to modulate vibration amplitude, the most significant feature of the results presented above is actually the distinct and separable changes in the amplitude and frequency outputs in response to input air pressure and motor power. This raises the possibility that the amplitude and frequency of the vibration response can be controlled independently by modulating the air pressure and motor signal respectively.

This result will form the basis of another study into the application of robust feedback control of both the vibration frequency and amplitude. The existing proportional controller worked surprisingly well under laboratory conditions, though it will be necessary to expand this to account for external influences and to eliminate the consistent error. Further optimisation may also improve the response time.

The observed behaviour under mechanical load is also an interesting result, which in itself raises the question of whether the user's manipulation could, consciously or unconsciously alter the intensity of felt vibration. This behaviour will need to be considered when designing a feedback controller as it will need to be able to correct for these effects. 
In the longer term, this technology presents itself as applicable to remote robot-environment interaction [11] and for haptic feedback in human-computer interaction [12] and virtual reality and wearable technology [13], [14].

\section{REFERENCES}

[1] G. Biroli, "A new kind of phase transition?" Nature Physics, vol. 3, pp. 222-223, 2007.

[2] M. Li, T. Ranzani, S. Sareh, L. D. Seneviratne, P. Dasgupta, H. A. Wurdemann, and K. Althoefer, "Multi-Fingered Haptic Palpation utilizing Granular Jamming Stiffness Feedback Actuators Smart Mater," Struct, vol. 23, p. 95007, 2014. [Online]. Available: http: //researchonline.rca.ac.uk/2973/1/SarehStiffnessFeedback2014.pdf

[3] A. A. Stanley, J. C. Gwilliam, and A. M. Okamura, "Haptic jamming: A deformable geometry, variable stiffness tactile display using pneumatics and particle jamming," 2013 World Haptics Conference, WHC 2013, pp. 25-30, 2013.

[4] A. A. Stanley, D. Mayhew, R. Irwin, and A. M. Okamura, "Integration of a Particle Jamming Tactile Display with a Cable-Driven Parallel Robot," in Haptics: Neuroscience, Devices, Modeling, and Applications, M. Auvray and C. Duriez, Eds. Springer Berlin Heidelberg, 2014, pp. 258-265.

[5] T. Sato, J. Pardomuan, Y. Matoba, and H. Koike, "ClaytricSurface: An Interactive Deformable Display with Dynamic Stiffness Control," IEEE Computer Graphics and Applications, vol. 34, no. 3, pp. 59-67, 2014.

[6] S. Follmer, D. Leithinger, A. Olwal, N. Cheng, and H. Ishii, "Jamming user interfaces: Programmable particle stiffness and sensing for malleable and shape-changing devices," UIST'12 - Proceedings of the 25th
Annual ACM Symposium on User Interface Software and Technology, pp. 519-528, 2012.

[7] Y. Kurihara, M. Koge, R. Okazaki, and H. Kajimoto, "Large-area tactile display using vibration transmission of jammed particles," IEEE Haptics Symposium, HAPTICS, vol. 1, no. I, pp. 313-317, 2014.

[8] I. Zubrycki and G. Granosik, "Novel Haptic Device Using Jamming Principle for Providing Kinaesthetic Feedback in Glove-Based Control Interface," Journal of Intelligent and Robotic Systems: Theory and Applications, vol. 85, no. 3-4, pp. 413-429, 2017.

[9] R. Rizzo, A. Musolino, and L. A. Jones, "Shape localization and recognition using a magnetorheological-fluid haptic display," IEEE Transactions on Haptics, vol. 11, no. 2, pp. 317-321, 2018.

[10] L. D. Goodfellow, "The sensitivity of various areas of the body to vibratory stimuli," Journal of General Psychology, vol. 11, no. 2, pp. 435-440, 1934

[11] I. Farkhatdinov, J.-H. Ryu, and J. Poduraev, "Control strategies and feedback information in mobile robot teleoperation," IFAC Proceedings Volumes, vol. 41, no. 2, pp. 14681-14686, 2008.

[12] B. Duvernoy, I. Farkhatdinov, S. Topp, and V. Hayward, "Electromagnetic actuator for tactile communication," in International Conference on Human Haptic Sensing and Touch Enabled Computer Applications. Springer, 2018, pp. 14-24.

[13] I. Farkhatdinov, N. Ouarti, and V. Hayward, "Vibrotactile inputs to the feet can modulate vection," in 2013 World Haptics Conference (WHC). IEEE, 2013, pp. 677-681.

[14] M. Ogrinc, I. Farkhatdinov, R. Walker, and E. Burdet, "Deaf-Blind Can Practise Horse Riding with the Help of Haptics," ser. Lecture Notes in Computer Science, F. Bello, H. Kajimoto, and Y. Visell, Eds. Cham: Springer International Publishing, 2016, vol. 9774, no. 37, pp. 452-461. 